\title{
The answer of the Bacteriology Laboratory to new clinical needs. Rapid sepsis diagnotics at the Novara hospital
}

\author{
Vesselina Kroumova, Elisa Gobbato, Paola Macaluso, Giacomo Fortina \\ Laboratorio di Microbiologia e Virologia Azienda Ospedaliera/Universitaria Maggiore della Carità Novara
}

Key words: Sepsi, TAT, Organizzazione del laboratorio, Emocoltura

La risposta del Laboratorio di Batteriologia alle nuove esigenze cliniche.

La Diagnostica rapida della sepsi presso I'A.O.U. “Maggiore della Carità” di Novara

\section{SUMMARY}

Faster microbiological responses are increasingly necessary in modern medicine and the Laboratory of Microbiology must be equipped in this sense.

New instrumentation and, above all, a new approach by the Clinical Microbiologist that puts a focus on the real needs of the patient before the microbiological may allow for significantly improving the TAT of these diagnostics. The use of both new methodologies, new tools and revisited old technologies may mean less these days as it was obtained at the Laboratory of Microbiology and Virology of Novara, where the combined use of molecular biology techniques, and mass spectrometry techniques rapid growth have allowed for more than 36 hours to shorten the response time by positivization of blood cultures.

Such an approach allows an important support to the clinician with obvious benefits for the patient.

La diagnostica microbiologica è sempre stata caratterizzata da tempi di risposta non rapidi, in particolare se paragonati a quelli abituali del Laboratorio di Chimica Clinica dove la rapidità delle risposte, insieme ad altri fattori, ha consentito una progressiva meccanizzazione delle analisi che, iniziata negli anni 70, è oggi alla base di una quasi completa automazione.

Il Laboratorio di Microbiologia, al contrario, pur molto cambiato rispetto a quegli anni, non ha subito una tale evoluzione limitandosi a pur importanti cambiamenti strumentali che, tuttavia, non hanno modificato significativamente $\mathrm{i}$ tempi di risposta (TAT $=$ turnaround time) che mantengono un'impostazione da "tempi del contadino" e, conseguentemente, sono caratterizzati dal tempo della semina, da quello dell'attesa e da quello dell'identificazione/raccolto.

Un importante cambiamento nei TAT del laboratorio di Batteriologia si è prospettato con l'avvento di nuove tecnologie, ad esempio la Biologia Molecolare. Questa tecnica permette di ridurre i tempi di molte diagnostiche microbiologiche consentendo la ricerca diretta del microrganismo ed eliminando in tal modo il tempo legato alla sua crescita. Tuttavia una tale opportunità è stata utilizzata soprattutto dalla diagnostica virologica che, attraverso tali metodiche, ha ridotto di molto i tempi di risposta, traendone l'ulteriore vantaggio di poter quasi completamente eliminare le lunghe procedure legate alle colture cellulari.

L'utilizzo di questa nuova tecnologia in Batteriologia è stato invece, almeno in un primo momento, limitato a ricerche sui microrganismi a crescita lenta o difficile come ad esempio micobatteri, legionelle, clamidie e pochi altri.

Per i microrganismi più tradizionali si son continuate ad utilizzare le normali procedure, a volte lievemente velocizzate grazie all'impiego di nuove strumentazioni, ma senza modifiche significative dei tempi di risposta. Lo scarso interesse iniziale da parte della diagnostica batteriologica per la Biologia Molecolare è derivato anche dalla considerazione che le metodiche tradizionali hanno già al loro interno un'amplificazione naturale: la crescita batterica su terreni di coltura che rende disponibili grandi quantità di microrganismo per le successive indagini.

Nel frattempo l'evoluzione della Medicina ha comportato cambiamenti importanti nelle esigenze dei pazienti, soprattutto per il notevole aumento di situazioni critiche per le quali i tempi di risposta non possono più essere quelli "del contadino". Buona parte dei pazienti sono soggetti immunodepressi per i quali le migliorate possibilità terapeutiche, l'allungamento della vita media, le nuove tecniche chirurgiche ed anestesiologiche oggi disponibili hanno consentito di migliorare la prospettiva di vita ma che, anche a causa delle diminuite difese immunitarie possono presentare

\section{Corresponding author: Vesselina Kroumova}

S.C.D.O. Laboratorio di Microbiologia e Virologia, A.O.U. "Maggiore della Carità"

Corso Mazzini 18 - 28I00 Novara - Tel.032I 3733595 - Fax 032I 3733588

E-mail: vesselina.kroumova@maggioreosp.novara.it 
numerosi problemi microbiologici. L'insieme di queste situazioni porta i colleghi clinici alla necessità di avere risposte rapide ed esaustive da parte del Laboratorio di Microbiologia.

A queste nuove esigenze cliniche il Laboratorio di Microbiologia, ed in particolare il settore Batteriologia, sta rispondendo con alcune difficoltà, poiché i miglioramenti tecnologici/strumentali non sono di per sé in grado di soddisfare in modo adeguato tali richieste.

Il Laboratorio di Microbiologia dell'Azienda Ospedaliera Universitaria di Novara ha tentato di dare una sua risposta al problema affrontandolo nel suo complesso e non solo da un punto di vista tecnologico.

Un primo aspetto che ci è sembrato essere a volte trascurato è la necessità di conoscere le vere esigenze del paziente e del clinico, non solo nel momento in cui la situazione è compromessa ma in precedenza, quando essa è ancora sotto controllo. Un'iniziativa in tal senso risulta molto impegnativa e, viste le risorse oggi disponibili, non può essere attuata in tutte le realtà. Si è pensato quindi di attivare un servizio di consulting, al momento limitato ai Reparti dove maggiore è la presenza di pazienti critici: quelli di Terapia Intensiva.

Per le analisi a loro destinate è stata predisposta una corsia privilegiata in modo da ridurre al minimo i tempi morti del percorso diagnostico. L'impegno si concretizza anche con la presenza al mattino di un microbiologo clinico presso questi Reparti, in modo da informare direttamente i colleghi clinici dei risultati ottenuti nel corso della notte, ma soprattutto per condividere con loro le successive scelte di indagini, sia in base ai risultati ottenuti che alle reali esigenze del paziente.

Tale impostazione implica un cambiamento radicale nella gestione degli esami. Questi infatti non sono più semplici accertamenti diagnostici richiesti al Laboratorio e a cui si deve dare una risposta corretta, ma diventano indagini volte a dare soluzioni ai problemi di un paziente di cui si conoscono i problemi e non solo quelli microbiologici.

È stato inoltre impostato un flusso di lavoro nel quale ogni passaggio avesse una sua logica e, nel limite del possibile, fosse in grado di fornire un'informazione utile al Clinico. Il migliore esempio a riguardo è certamente l'approccio alla diagnostica della sepsi. È infatti dimostrato come per questi pazienti una terapia inadeguata determina un aumento della mortalità del $7 \%$ per ogni ora di ritardo (1). È quindi evidente come, proprio in casi di questo tipo, un'adeguata organizzazione del lavoro sia importante per fornire risultati clinicamente utili nel più breve tempo possibile.

Il TAT medio per la refertazione di un'emocoltura (BC), completa di identificazione e test di sensibi- lità, utilizzando le tecniche tradizionali si aggira intorno alle 54/60 ore, considerando che in questi pazienti il tempo di positivizzazione dell' $80 \%$ delle $\mathrm{BC}$ è compreso fra le 12 e le 18 ore (dati personali non pubblicati).

Bisogna sottolineare che la definizione del Tempo 0 di prelievo in un Laboratorio come il nostro nel quale non è attivo un servizio h24 non risulta agevole e rende anche la temporizzazione dei passaggi successivi non di facile comprensione. Per semplificare tutto ciò si è pensato di indicare nel testo come Tempo 0 le ore 8 del mattino successivo alla positivizzazione della $\mathrm{BC}$ ossia l'inizio, per la maggior parte di queste, delle procedure atte all' $i$ dentificazione e ai test di sensibilità nei confronti del microrganismo responsabile della sepsi. Questo perchè 1'organizzazione che noi abbiamo attuato, con l'eccezione del LightCycler ${ }^{\circledR}$ SeptiFast Test MGRADE, si pone l'obiettivo di diminuire i tempi di risposta dal momento della positivizzazione della $\mathrm{BC}$.

Considerando che in un Laboratorio come il nostro molte di queste positività si rilevano nella prima mattina del giorno successivo al prelievo e, in particolare, dopo l'incubazione notturna abbiamo pensato di organizzare la nostra attività in base alla concentrazione di campioni positivi presenti nelle prime ore della mattinata.

Per la lavorazione delle $\mathrm{BC}$ nel nostro laboratorio sono presenti due differenti percorsi diagnostici, contemporanei ma indipendenti. Il primo è quello tradizionale (Figura I), che porta ad una risposta completa dopo circa 3 giorni dall'inoculo del flacone dell'emocoltura. Il secondo tende ad accelerare le indagini di identificazione e i saggi di sensibilità e ci porta a disporre dei risultati entro sei ore dall'avvenuta positivizzazione dell'emocoltura (Figura II).

A quest'ultimo percorso fa riferimento anche la ricerca di microrganismi nel sangue mediante Biologia Molecolare che da noi viene eseguita mediante LightCycler ${ }^{\circledR}$ SeptiFast Test MGRADE (Roche Diagnostics) metodica che è in grado di identificare oltre 20 microrganismi fra quelli più frequentemente responsabili di sepsi.

Le procedure eseguite per questo test sono effettuate secondo le indicazioni della ditta produttrice. L'analisi consente, nei casi di positività, di giungere ad identificare il microrganismo responsabile dell'infezione in meno di sei ore dall'arrivo del campione di sangue in laboratorio.

Considerando che, ad oggi, il test di riferimento per questa ricerca rimane la coltura tradizionale e che l'indagine molecolare non è in grado, identificando solo materiale genetico, di dare indicazioni per i saggi di sensibilità, a questa indagine è sempre affiancata la ricerca colturale. Da notare 
inoltre che, da noi, questo test è effettuato solo dopo consulto fra clinico e microbiologo clinico. La presentazione che segue illustrerà essenzialmente i passaggi legati alla via accelerata considerando quelli della via tradizionale come ormai consolidati.

Per la BC positiva viene subito allestita la colorazione di Gram che consente di definire caratteristiche tintoriali e morfologiche delle cellule batteriche presenti. Ovviamente queste informazioni vengono immediatamente riportate e valutate con $\mathrm{i}$ colleghi clinici. Successivamente in base a queste caratteristiche viene eseguito, direttamente dalla $\mathrm{BC}$ positiva, un test di sensibilità mediante ETest ${ }^{\circledR}$ (Bio-test) per i seguenti antibiotici precedentemente concordati con i clinici di quei Reparti dove è attivo il servizio di consulting. Per microrganismi Gram positivi: Vancomicina, Teicoplanina e Oxacillina. Per microrganismi Gram negativi: Ricerca ESBL, Piperacillina/Tazobactam, Levofloxacina, Amikacina e Imipenem. In situazioni particolari l'elenco degli antibiotici può essere modificato. Questo passaggio consente di disporre, a volte già nel tardo pomeriggio dello stesso giorno di positivizzazione della $\mathrm{BC}$, più spesso la mattina successiva, di un dato certo di sensibilità nei confronti degli antibiotici più utilizzati dai clinici. L'esecuzione dell'ETest ${ }^{\circledR}$ consente inoltre di disporre anche della MIC, informazione che, in questi pazienti, risulta spesso fondamentale.

Un'ulteriore aliquota della BC positiva viene trattata e incubata nello strumento HB\&L (Alifax) con una metodica home-made (5). Un tale passaggio, oltre a consentire di operare successivamente con una concentrazione batterica maggiore e standardizzata, permette la diluizione di eventuali sostanze inibenti presenti nei flaconi della $\mathrm{BC}$ (resine, carbone, antibiotici, etc...) con conseguente migliore resa $(3,4)$.

Quando lo strumento HB\&L segnala il raggiungimento di una concentrazione di $0.5 \mathrm{McF}, 1 \mathrm{~mL}$ di brodo viene utilizzato per procedere all' $i-$ dentificazione del microrganismo presente mediante lo spettrometro di massa MALDI-TOF (Bruker) seguendo la metodica da noi messa a punto (3). Sempre dalle vials dello strumento $\mathrm{HB} \& \mathrm{~L}$ vengono prelevati $100 \mu \mathrm{L}$ di tale coltura per ognuno degli antibiotici da saggiare ed aggiunti a vials contenenti brodo addizionato ad una quantità nota dell'antibiotico. Tale impostazione consente di avere identificazione batterica e, seppur preliminarmente, informazioni sulla sensibilità del ceppo in esame nei confronti degli antibiotici saggiati già dopo solo $4 / 6$ ore dall'avvenuta positivizzazione della BC (Figura II) con risultati la cui validità è già stata oggetto di pubblicazione $(3,4,7)$. In particolare l'identificazione dei microrganismi mediante spettrometria di massa MALDI-TOF direttamente da $\mathrm{BC}$ positive, come da noi già pubblicato, ha mostrato concordanza assoluta a livello di genere e del $97.5 \%$ a livello di specie nei confronti delle stesse identificazioni effettuate mediante Vitek-2® (bioMérieux) (3). Anche i dati relativi ai test di sensibilità hanno mostrato buona riproducibilità nei confronti di ETest ${ }^{\circledR}$ (Bio-test) soprattutto per quanto riguarda i microrganismi Gram positivi (7).

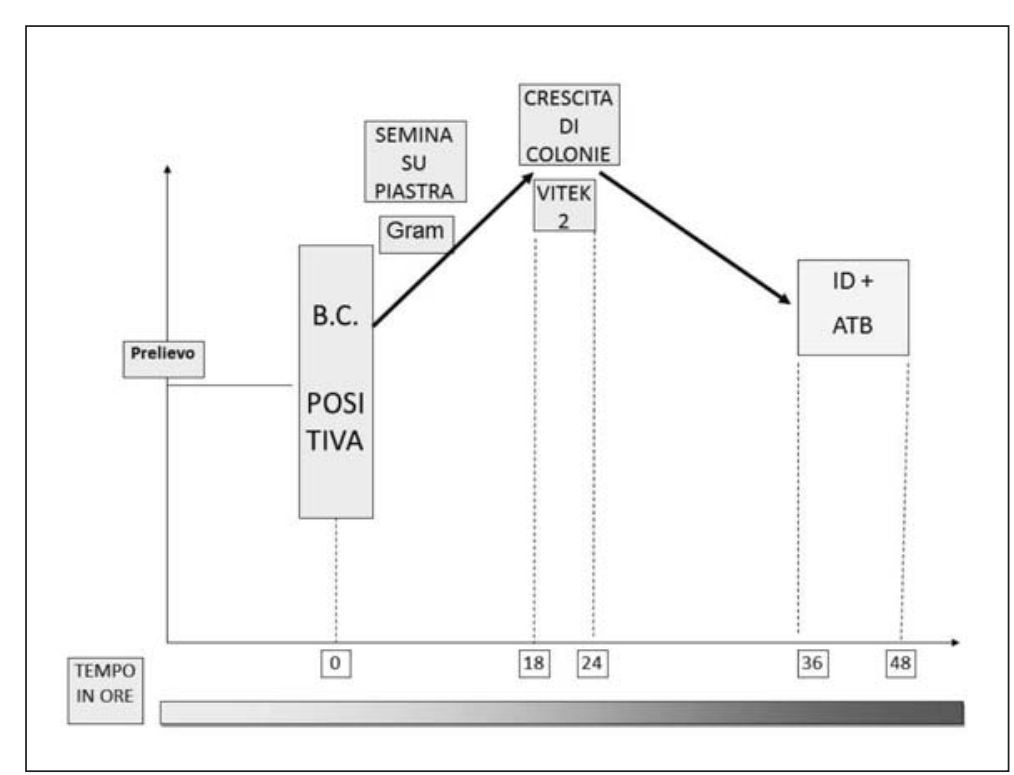

Figura I. BC: flusso di lavoro tradizionale.

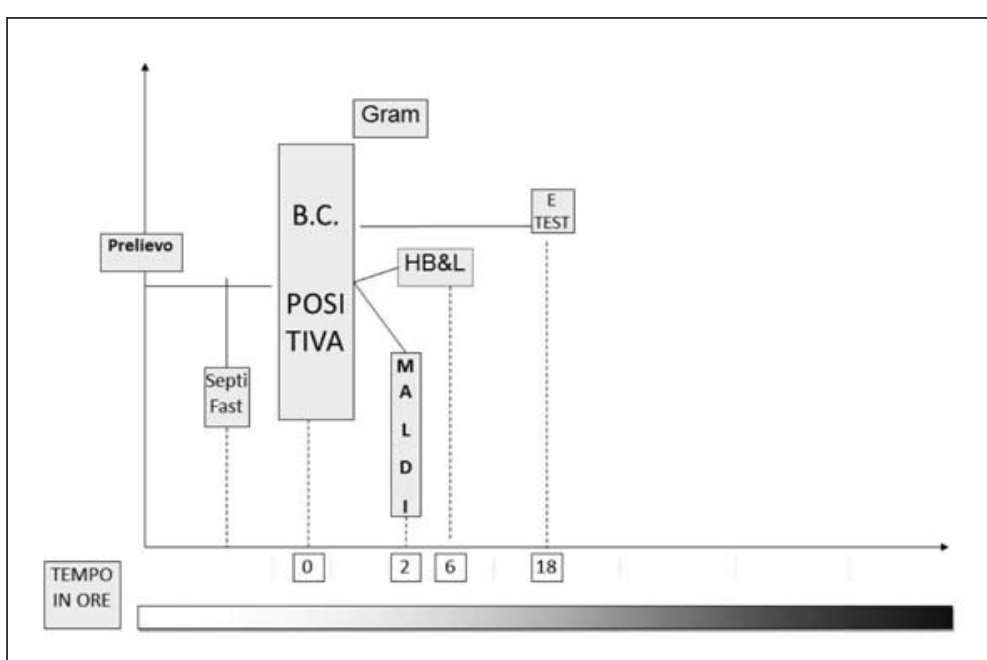

Figura II. BC: flusso di lavoro accelerato. 
Grazie a questa organizzazione di lavoro, siamo riusciti ad ottenere risposte complete, identificazione ed antibiogramma, in un tempo che consente un intervento terapeutico mirato molto più precocemente rispetto a quanto possibile con le metodiche tradizionali.

Riteniamo che un tale risultato, raggiunto grazie all'utilizzo di metodiche sia innovative che tradizionali ma pensate con un'impostazione di lavoro dedicata alle reali esigenze del paziente, ci consenta di offrire al clinico dati microbiologici completi con circa due giorni di anticipo rispetto alla normale pratica batteriologica.

Un tale risultato, pur non consentendo ancora l'inizio di una terapia mirata già dai primi sintomi della sepsi, consente di modificare con largo anticipo un'eventuale terapia non corretta o di effettuare una de-escalation con indubbi vantaggi sia per il paziente che per l'epidemiologia dell'antibiotico resistenza $(1,2)$.

Infine possiamo affermare che questa nostra organizzazione di lavoro dimostra come, grazie ad una maggior attenzione alle esigenze del clinico e soprattutto del paziente, sia possibile offrire dati microbiologici in tempi significativamente più brevi rispetto alle metodiche tradizionali grazie ad una organizzazione di lavoro volta più al vantaggio del paziente che all'immediata completezza del dato microbiologico.

\section{BIBLIOGRAFIA}

1. Ellington MJ, Bentley N, Pai S, et al. MALDI-TOF identification from BacT/Alert blood culture in Cambridge, UK. 2011. 21 $1^{\text {st }}$ European Congress of Clinical Microbiology and Infectious Diseases (ECCMID) Abstract id: 124445.

2. Haigh JD, Ball D, Eydmann M, Millar M, Wilks M. Direct identification of microorganisms from the bioMérieux BacT/Alert blood culture system by MALDI-TOF is possible. 2011. 21 ${ }^{\text {st }}$ European Congress of Clinical Microbiology and Infectious Diseases (ECCMID). Abstract id: 128162.

3. Kroumova V, Gobbato E, Basso E, Mucedola L, Giani T, Fortina G. Direct identification of bacteria in blood culture by matrix-assisted laser desorption/ionization time-of-flight mass spectrometry: a new methodological approach. Rapid Commun Mass Spectrom. 2011; 25(15): 2247-9.

4. Kroumova V, Gobbato E, Giani T, et al. Direct identification from BacT/Alert ${ }^{\mathrm{TM}}$ blood culture bottles using MALDI-TOF.(in press).

5. Kroumova V, Gobbato E, Macaluso $P$, et al. Preliminary indications for antibiotic susceptibility tests in less than six hour in positive blood cultures. Microbiologia Medica 2010; 25(1): 24-6.

6. Kumar A, Roberts D, Wood KE, et al. Duration of hypotension before initiation of effective antimicrobial therapy is the critical determinant of survival in human septic shock. Crit Care Med. 2006 Jun; 34(6): 1589-96.

7. Olivieri C, Vaschetto R, Kroumova V, et al. Rapid assessment of antibiotic-susceptibility in positive blood culture. Poster from 23rd Annual Congress European Society of Intensive Care (ESICM) Oct 913, 2010 Barcelona. 